\title{
Light microscopy of Indian ink preparations of fibrillated cartilage
}

\author{
G. MEACHIM
}

Department of Pathology, University of Liverpool

At present it is not known whether fibrillation of articular cartilage is initiated by abrasive or adhesive wear, by fatigue failure (Freeman, 1971) or overstressing, or by some other process such as the 'sliding damage' postulated by Walker, Sikorski, Dowson, Longfield, Wright, and Buckley (1969); moreover, it cannot be assumed that each instance of surface damage is always initiated in the same way. Morphological observations are relevant to this problem, since it is important that any hypothesis eventually put forward to explain fibrillation should be fully consistent with structural changes observed in the tissue. Previous studies have described the morphology of fibrillation as seen by light microscopy of conventional histological sections cut vertical to the articular surface (Collins, 1949; Meachim, Ghadially, and Collins, 1965); as seen by transmission electron microscopy of ultrathin vertically cut sections (Meachim and Roy, 1969); as seen by scanning electron microscopy of the cartilage viewed from above the surface (McCall, 1968; Walker and others, 1969; Redler and Zimny, 1970); and as seen by macroscopic examination of Indian ink preparations (Bullough and Goodfellow, 1968). The present study employs the use of methods in which Indian ink preparations are examined by reflected light microscopy of the surface in situ and by transmitted light microscopy of tangential surface slices.

\section{Materials and methods}

The study was made on the patello-femoral articulation of one knee joint, usually the left, from an unselected series of necropsies in 29 subjects ( 21 men and 8 women) whose ages ranged from 18 to 91 years; joints showing changes from inflammatory disease or disuse atrophy were

Accepted for publication May 1, 1972 excluded. The specimens were examined as soon as possible during or after the necropsy.

\section{Indian ink preparations}

The cartilage surface was rinsed with physiological saline, and then painted with Indian ink diluted in saline. Excess ink was removed by gentle wiping with moistened cotton wool or lens tissue. Before and after painting, the cartilage was kept wet by the use of physiological saline; no fixative was used. The painted surface of the cartilage, in situ on its underlying bone, was examined en face at a magnification of $\times 10$, using a 'Stereomaster' microscope (W. B. Prior) with reflected light from the lamp mounted in the focusing holder.

\section{Artificial splits}

The point of a pin was used to make artificial splits into the cartilage surface (Bullough and Goodfellow, 1968). For photography, the splits were filled with Indian ink.

\section{Tangential slices}

Thin tangential slices of the Indian ink preparations were cut from the surface of the unfixed wet tissue, using singleedge safety razor blades manipulated under the stereomicroscope. The slices were mounted in physiological saline on glass slides without coverslips, and examined by light microscopy using transmitted light. The preparations were studied unstained except for the Indian ink particles present on their surface.

\section{Vertical sections for histology}

Tissue blocks for histology were taken from some of the Indian ink preparations. A note was made of the alignment of the block in relation to the direction of the proximaldistal axis of the articulation and to the direction taken by the artificial splits. The blocks were fixed in buffered formol-saline, processed, embedded in paraffin wax, and cut vertical to the articular surface. Sections were examined unstained except for the Indian ink particles on the surface. 


\section{Results}

SURFACE APPEARANCE IN THE TANGENTIAL PLANE

The appearance of the surface, when examined en face in the tangential plane, varied from specimen to specimen and from site to site on the same specimen. Taking the series as a whole, four main types of surface appearance were encountered (Figs 1 to 7) in the Indian ink preparations.

All the illustrations are of Indian ink preparations unless indicated otherwise. All the specimens are from the patellofemoral articulation in an unselected series of necropsies.

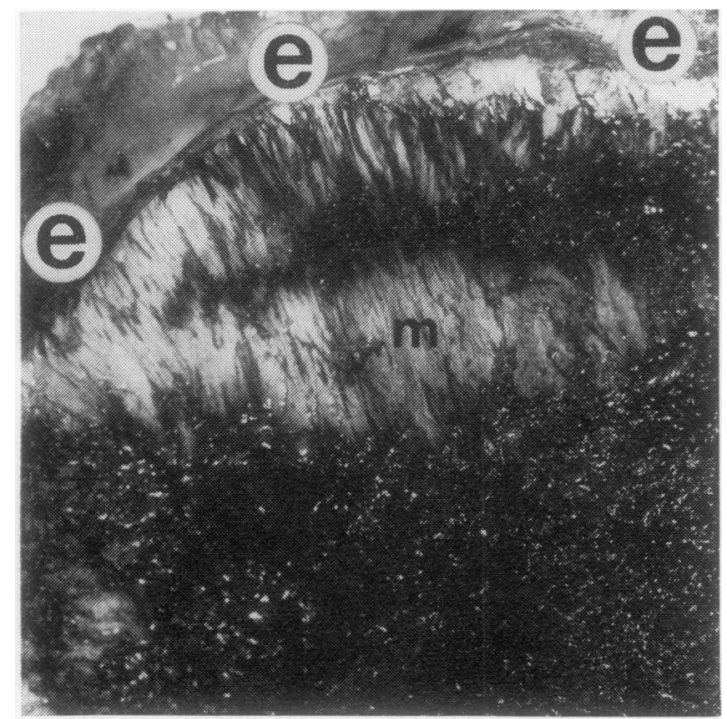

FIG. 1 Part of a patellar articular surface, viewed en face in reflected light. Man aged 70 years. In this specimen most of the cartilage shows a confluent blackening indicative of 'overt fibrillation'; in contrast, surface areas of 'minimal fibrillation' ( $m$ ) show dark markings against a pale grey background. Patellar edge $(e) . \times 3$

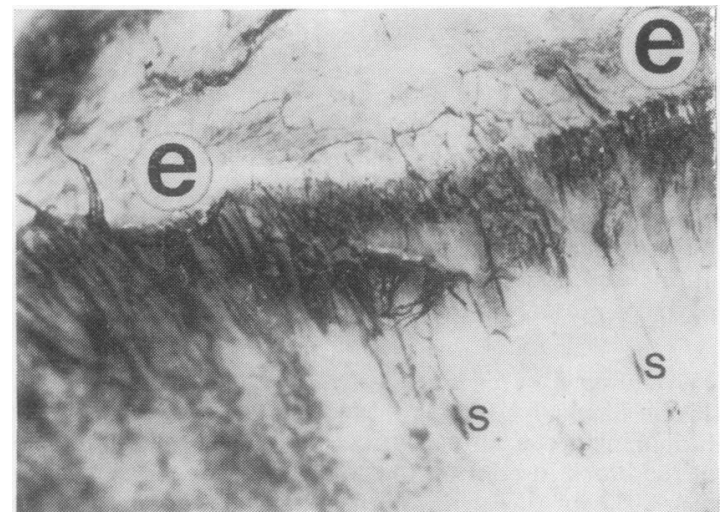

FIG. 2 Part of a patellar surface viewed en face in reflected light. Man aged 48 years. Semi-confluent blackening, with flakes of partly loose tissue, is seen near the edge (e) of the joint surface. Artificial splits $(S) . \times 3$
(3)

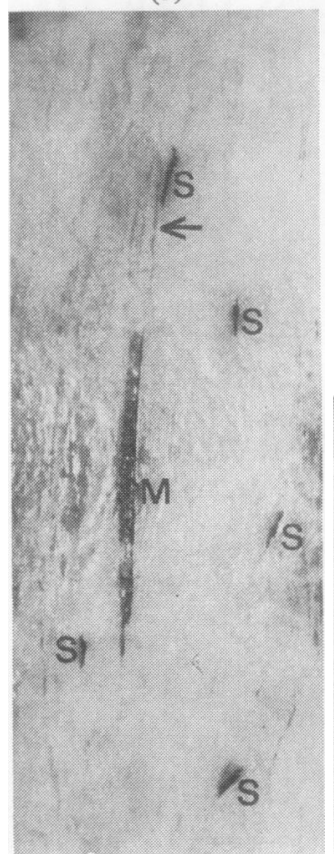

FIG. 3 A more central, femoral, surface of the joint illus trated in Fig. 2. In this field the surface mostly appeate 'intact' apart from the presence of some fine marking (arrow) and of one broader marking $(M)$. In this pattern of 'minimal fibrillation' the markings show the same, predominantly proximal-distal, orientation as elongated splits $(S)$ which have been produced artificially by pricking the surface with the rounded point of a pin. $\times 3$

FIG. 4 Part of a patellar articular surface viewed en face in reflected light. Man aged 26 years. Although most of this patellar surface was 'intact', there were also some fine markings and occasional, partly loose flakes and strands indicative of 'minimal fibrillation'. The long narrow strand of tissue $(N)$ was loose except for an attachment at one end, near the proximal edge (e) of the joint surface; it originally lay in a proximal-distal alignment, and has been deliberately shrunken and curled to facilitate photography. Artificial splits $(S) . \times 3$

FIG. 5. Part of a patellar articular surface viewed en face in reflected light directed from an angle. Man aged 61 years. A feature which appears to be a sharply-defined shallow groove, with a highlight along one border, is present at the upper and central right of the field. Fine and broader linear markings are also seen. In this pattern of 'minimal fibrilla tion' the markings have a predominantly parallel alignment $\times 16$

(1) Sites at which no distinct surface markings on the cartilage were seen on stereomicroscopy at a magnification of $\times 10$. Such sites were usually found also to have an unmarked appearance if subsequently examined in tangential slices at a magnification of $\times 150$. These surfaces can be termed 'intact'. A pin 


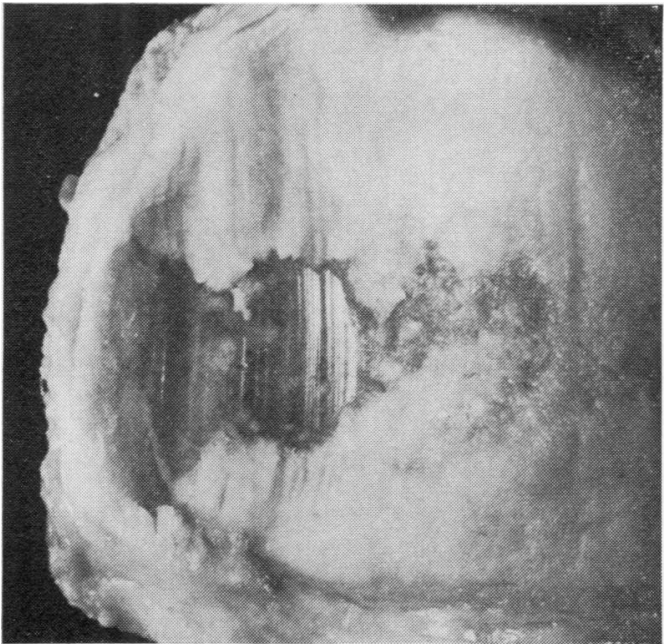

FIG. 6 Patellar surface viewed en face in reflected light, before painting with Indian ink. Woman aged 71 years. On the left there is an area of full-thickness loss of cartilage with exposure of the underlying bone. Most of the patellar cartilage which still remains shows overt fibrillation with grooves in a proximal-distal alignment; grooving in the same alignment is seen on the exposed bone surface (linear highlights). $\times 1$

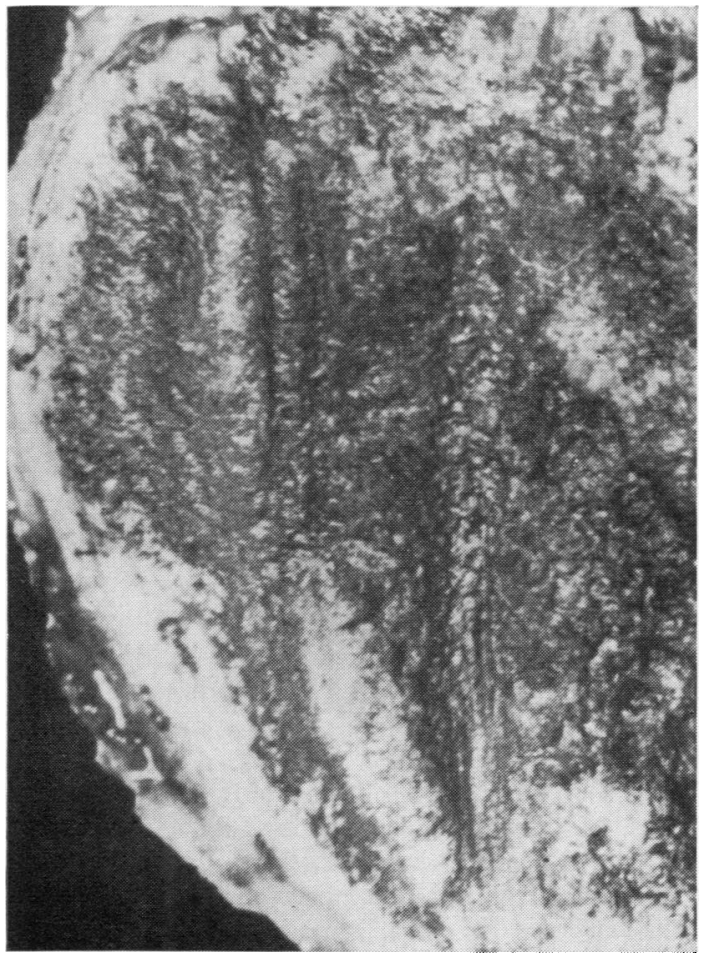

FIG. 7 Overtly fibrillated patellar cartilage showing grooving in a proximal-distal alignment. Woman aged 82 years. Similar grooving was seen on the opposing femoral cartilage. No bone exposure was present at this patellofemoral articulation. $\times 4$ prick into an 'intact' site formed an elongated split (Figs 2, 3,4) in the superficial zone of the cartilage. The artificial splits usually formed in a similar direction to that of the proximal-distal axis of the articulation, although deviations from this direction can occur near the medial or lateral edge of the joint surface.

(2) Sites showing dark markings against a pale grey background (Figs 1,3) when examined by stereomicroscopy and in tangential slices (Fig. 8). This appearance can be termed 'minimal fibrillation'.

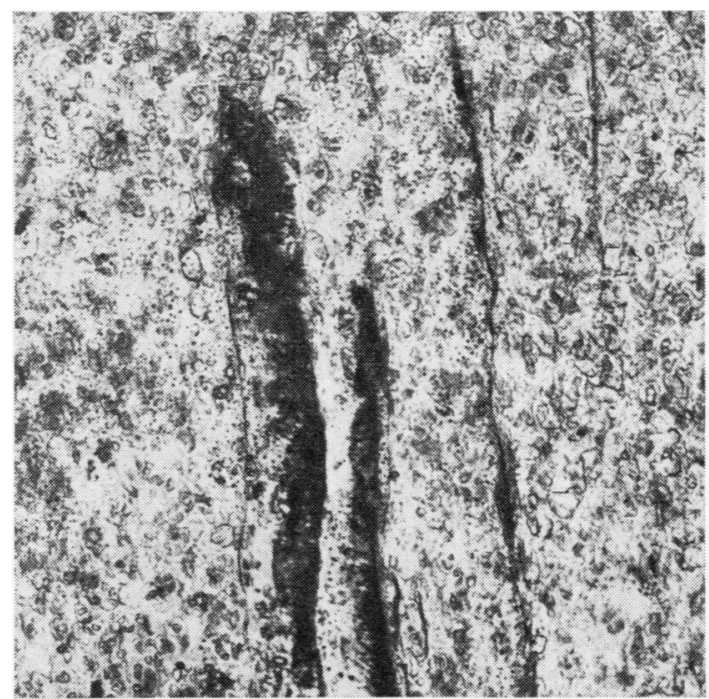

FIG. 8 Tangential surface slice of unfixed tissue, mounted in physiological saline, viewed by transmitted light. Fine or broad dark markings are seen against a background texture which represents the underlying cells (Fig. 9). In the pattern of 'minimal fibrillation' shown here the markings are elongated and in approximately parallel alignment. Femoral surface. $\times 60$

Tangential slices of such surfaces (Fig. 8) showed dark, fine or broad, markings when examined by transmitted light at a magnification of $\times 150$ (Figs 10 , $11,12)$, contrasting with the unmarked appearance of sites which appeared 'intact' in tangential slices (Fig. 9). These markings were seen against a background texture from the presence of cells in the tissue beneath the surface (Figs 8, 9). The broader markings sometimes had a curled uneven appearance along one edge (Fig. 10) and examination of slices mounted without a coverslip showed that this appearance represented tissue partly loosened from the surface by a process of splitting occurring at the microscopic level; a grosser version of the splitting process had in some instances led to the formation of partly loosened flakes or strands readily apparent to the naked eye (Fig. 4).

The markings found at sites of 'minimal fibrillation' exhibited a variety of patterns when examined in the tangential plane. Two main groups of patterns 


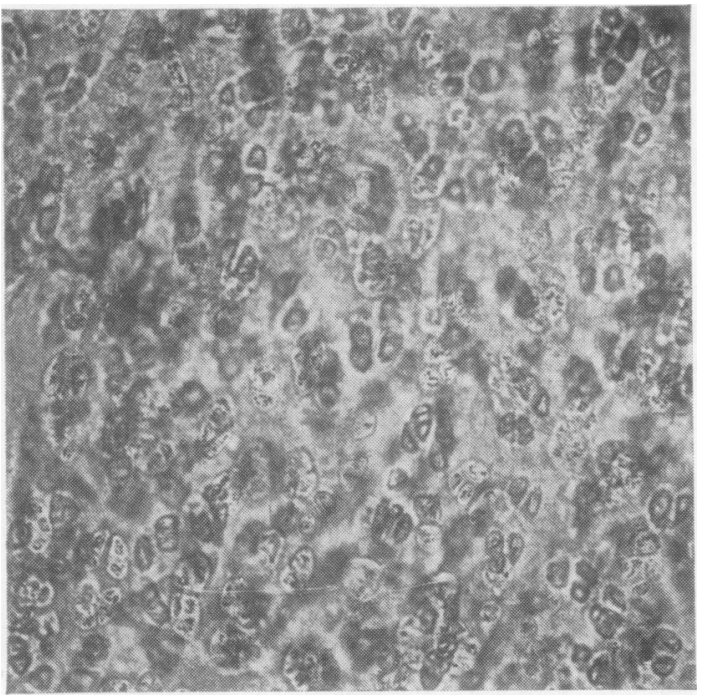

FIG. 9 Tangential slice of 'intact' unmarked surface, showing the background texture from the underlying cells when viewed in transmitted light. $\times 150$

Table Age of the subjects in relation to the percentage of the total area of the patellar surface showing 'overt' fibrillation, to the percentage of the patellar surface showing bone exposure, and to the presence or absence of patellar marginal osteophytes

\begin{tabular}{|c|c|c|c|}
\hline $\begin{array}{l}\text { Age } \\
\text { (yrs) }\end{array}$ & $\begin{array}{l}\text { 'Overt' } \\
\text { per cent. } \\
\text { fibrillation }\end{array}$ & $\begin{array}{l}\text { Per cent. } \\
\text { bone } \\
\text { exposure }\end{array}$ & $\begin{array}{l}\text { Patellar } \\
\text { osteophytes }\end{array}$ \\
\hline 18 & 19 & 0 & - \\
\hline 21 & 27 & 0 & - \\
\hline 22 & 0 & 0 & - \\
\hline 22 & 0 & 0 & - \\
\hline 23 & 0 & 0 & - \\
\hline 26 & 38 & 0 & - \\
\hline 31 & 10 & 0 & - \\
\hline 40 & 18 & 0 & - \\
\hline 44 & 24 & 0 & - \\
\hline 44 & 94 & 0 & - \\
\hline 46 & 20 & 0 & - \\
\hline 48 & 50 & 0 & - \\
\hline 48 & 52 & 0 & - \\
\hline 49 & 42 & 0 & - \\
\hline 52 & 40 & 0 & - \\
\hline 56 & 22 & 67 & + \\
\hline 59 & 89 & 0 & - \\
\hline 60 & 60 & 0 & - \\
\hline 60 & 76 & 0 & - \\
\hline 61 & 96 & 0 & - \\
\hline 70 & 83 & 0 & - \\
\hline 70 & 70 & 0 & - \\
\hline 71 & 81 & 19 & + \\
\hline 72 & 25 & 0 & - \\
\hline 78 & 100 & 0 & - \\
\hline 81 & 96 & 0 & - \\
\hline 82 & 88 & 0 & - \\
\hline 84 & 83 & 0 & - \\
\hline 91 & 57 & 43 & + \\
\hline
\end{tabular}

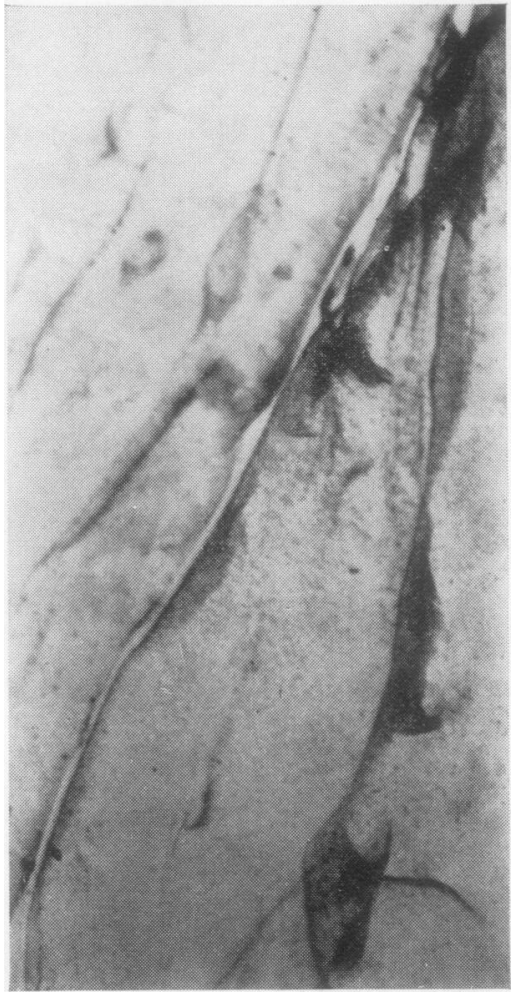

FIG. 10 Tangential surface slice of unfixed tissue, viewe $\vec{t}^{2}$ by transmitted light adjusted to obscure the background texture from the cells. 'Minimal fibrillation' showing dark markings; some of the broader markings have a curled uneven appearance along one edge, and represent tissue partly loosened from the surface. Mounted in saline without a coverslip. Patellar surface. $\times 150$

were noted. In one the markings showed approximately parallel alignments (Figs 8, 10), usually running in the same direction as that taken by nearby artificial splits (Fig. 3); sites with this pattern sometimes showed long, narrow or broad, strands of partly loosened tissue (Fig. 4), and sharply-defined grooves were sometimes apparent (Fig. 5). In the second group of patterns, the Indian ink markings show various other more complex configurations (Figs 11, 12) and alignments; one common example from amongst this group has a reticular, mosaic-like appearance (Fig. 12).

(3) Sites showing semi-confluent (Fig. 2) or confluent (Fig. 1) blackening of the cartilage. This appearance was in most instances indicative of 'overt', fullydeveloped, fibrillation of exposed cartilage. However, at the extreme edge of the joint, it was found by subsequent histology that blackening could be due either to overt fibrillation or to an encroachment of a layer of fibrous tissue over the cartilage surface. Grooving in the proximal-distal axis was sometimes seen on overtly fibrillated cartilage (Figs 6, 7), and on 


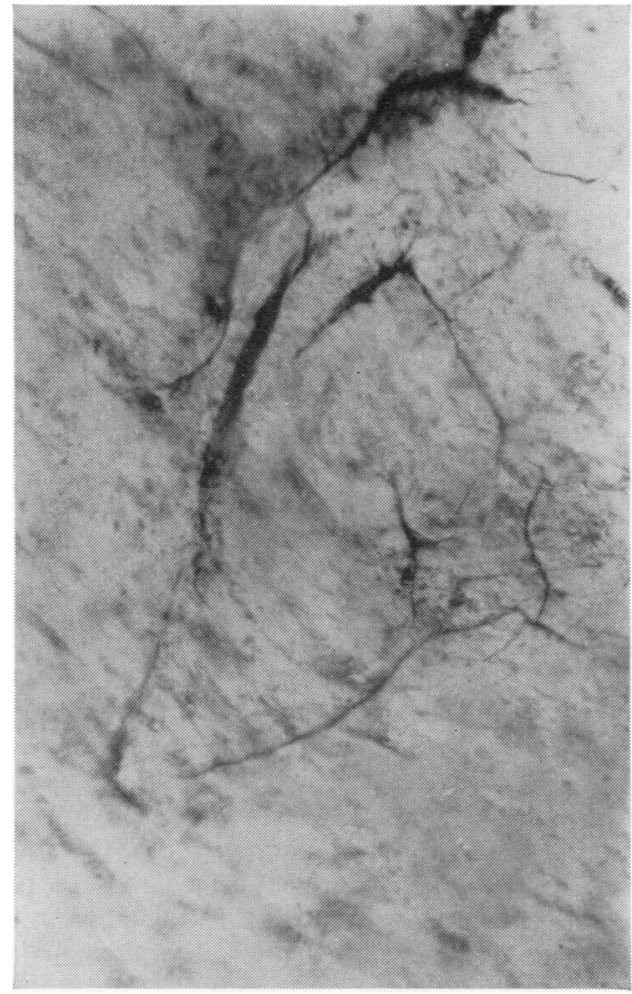

FIG. 11 'Minimal fibrillation', showing fine and broader markings which in this field form a more irregular pattern than that seen in Figs 8, 10. Tangential slice, unfixed tissue. Femoral surface. $\times 150$

adjacent bone if this had become exposed (Fig. 6). In one patellar specimen, a grooved area of thinned cartilage on which the superficial layer had been destroyed showed less than the expected amount of blackening; histologically, the affected area was found to have a relatively smooth surface without the deep splitting usually seen in overt fibrillation.

(4) Sites showing full-thickness loss of cartilage, with exposure of underlying bone (Fig. 6).

\section{QUANTITATIVE RESULTS}

For each of the specimens, a point-counting technique was used to estimate the amounts, if any, of 'overt' fibrillation and of bone exposure on the patellar surface; the results were expressed as percentages of the total surface area of the patellar articular face (Table). A note was also made of the presence or absence of patellar osteophyte formation. The amount of overt fibrillation tended to increase with age (Table). In younger subjects, overt fibrillation, if present, was seen particularly at or near the edges of the patellar surface (Fig. 2) and on its medial facet; in many older subjects most or all of the surface was affected, and it would appear that no anatomical site on the patella is immune from cartilage damage by this age-related process. The results are in keeping

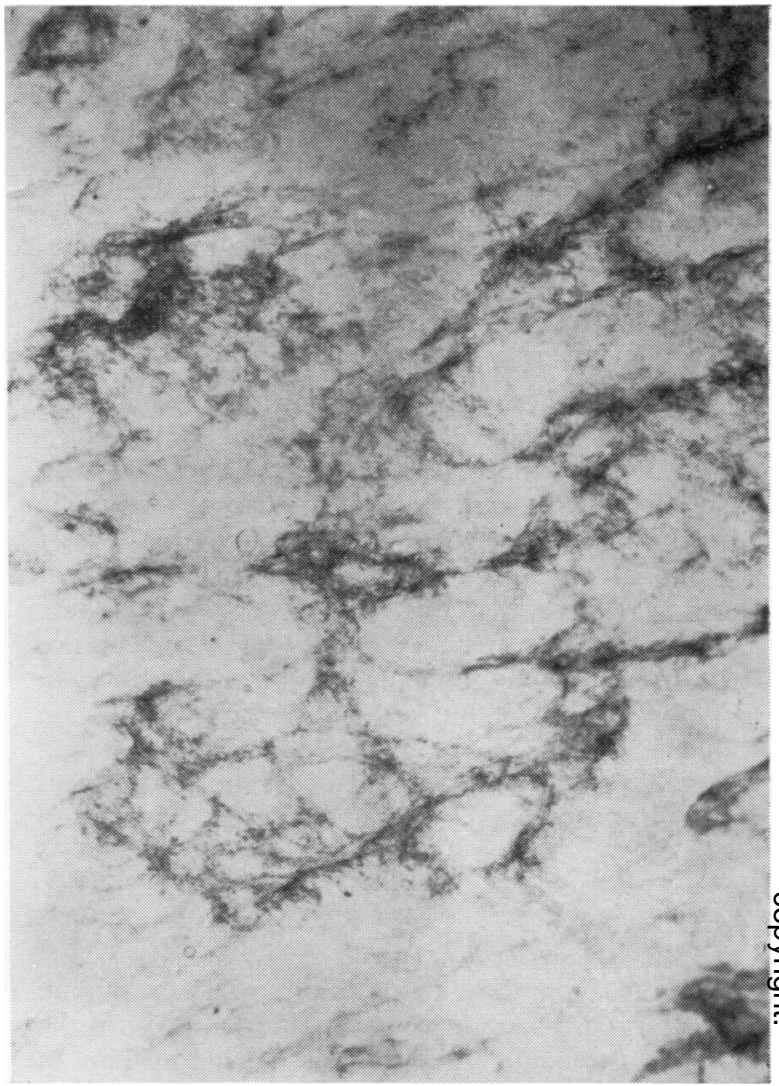

FIG. 12 A mosaic-like reticular pattern commonly encountered at patello-femoral sites showing 'minimal fibrillation' (compare with Fig. 10). Tangential slice, unfixed tissue. Femoral surface. $\times 150$

with previous observations by Bennett, Waine, and Bauer (1942). Further quantitative and anatomical studies of the patellar and opposing femoral surface are at present in progress (Emery and Meachim, unpublished observations).

SURFACE APPEARANCE IN THE VERTICAL PLANE No exact correlation is claimed between the terminology used in describing the tangential slices and the appearances seen in vertically cut histological sections. The morphological appearance of the articular surface was influenced by which type of preparation was examined and by which particular field was actually under the microscope. Moreover the alteration in morphology from an 'intact' surface through 'minimal' to 'overt' fibrillation represented, on the microscopic scale, a patchy process.

A surface which appeared locally intact in a vertically cut histological section examined at a magnification of $\times 150$ presented a particle-covered line free from sharply-angled irregularities in the particular microscopic field under study (Fig. 13 overleaf). 
FIG. 13 Histological section of fixed tissue, cut vertically, and showing in this particular microscopic field an 'intact' surface on which the line of pigment shows no sharply-angled irregularities (compare with Figs 14, 15) when examined at a magnification of $\times 150$. In this and all subsequent illustrations the synovial space is at the upper part of the field, with tissue below.

Deviations from this intact pattern (Figs 14 to 17, opposite) showed minute, sharply-angled irregularities; splitting immediately beneath the surface from one edge of such an irregularity; tissue free at one margin but still elsewhere in continuity with the rest of the cartilage; and cavities within the tissue immediately beneath the surface. Other patterns were also encountered (Figs 18 and 19). The appearance was influenced by the alignment of the block in relation to that of the surface markings shown on tangential slices (Figs 14 to 17 and 20). These minimal morphological changes merged into the appearances seen in histological sections of overt fibrillation (Fig. 21).

\section{Discussion}

By use of Indian ink preparations when examining articular cartilage, areas of mild damage to the surface are made more readily apparent than in unstained preparations. The findings indicate that areas of the surface in this state of 'minimal fibrillation' can exhibit a variety of patterns when examined en face by stereomicroscopy and by transmission light microscopy of tangential slices. With the techniques described, these various patterns can be studied on cartilage which is unfixed and in contact with physiological saline, thus avoiding artefactual distortions from the shrinkage and edge curling which is said to occur during preparation of specimens for scanning electron microscopy (Clarke, 1971). As an incidental advantage, Indian ink preparations also aid in the interpretation of histological sections cut after fixation of the tissue, since the particles mark the line of the articular surface and enter cavities which were in communication with the synovial space; thus the Indian ink helps to distinguish between genuine and artefactual irregularities of the surface. The present investigation further shows that the appearance of the surface as seen in vertical sections can be influenced by the direction in which the block is cut in relation to the alignment of surface features (Figs 14 to 17 and 20).

The results of the present study suggest that one way in which the state of 'minimal fibrillation' may be initiated is from the sites of a feature seen in vertical sections as minute, sharply-angled irregularities of the line of the articular surface (Figs 14, 15); this feature is apparent on light microscopy at a magnification of $\times 150$. The fine dark markings seen on tangential slices of Indian ink preparations (Figs 8, 10, 11,12 ), examined at the same magnification, evidently represent the same feature: because of the semitranslucency of the tissue, multilayering of the ink particles due to infolding of the surface line at the site of the irregularity will appear as a localized concentration of pigment when the surface is viewed from above; entrapment of particles in the crevice may contribute to the visual effect. The suggestion that the markings are due to 'ink trapped by revealed fibres' (Bullough and Goodfellow, 1968) is probably also valid, but does not seem to be the complete explanation of the phenomenon. In this context it is relevant that microscopy shows that the particles also adhere to 'intact' surfaces (Fig. 13) and not just to the sites of surface irregularities.

The methods used virtually exclude the possibility that these minute surface irregularities, seen at a magnification of $\times 150$, are technical artefacts. From one edge of the initial irregularity, splitting extends immediately beneath the surface (Figs 14 to 17), giving a feature represented by broader dark markings on tangential slices (Figs 8, 10, 11). At certain levels of vertical section, the splitting process is seen as a cavity within the tissue (Fig. 16); such cavities contain Indian ink particles, a finding which suggests that they communicate elsewhere with the synovial space; the superficial 'blisters' which have been observed on transmission electron microscopy (Meachim and Roy, 1969) may be an ultrastructural equivalent of the same phenomenon. The tangential orientation of the splits is probably a consequence simply of the tangential orientation of the superficial collagen fibres, since the cartilage tends to split along the plane of the fibre network (Collins, 1949). As a result of the splitting process, pieces of cartilage become free along one or more of their edges while elsewhere still remaining in continuity with the rest of the surface tissue (Figs 15, 17). Partly loosened flakes and strands, of various shapes, can be produced in this manner.

The sequence of events postulated above will account for 'minimal fibrillation' surface patterns of the type in which the Indian ink markings tend to show approximately parallel alignments when viewed 


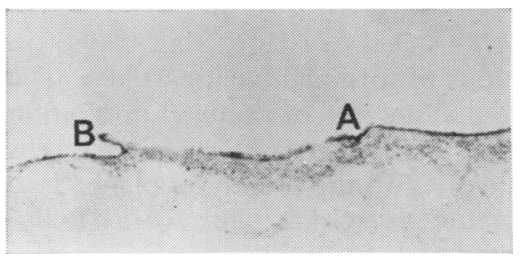

(14)

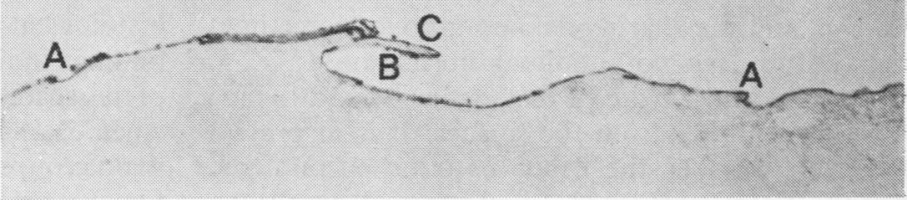

(15)

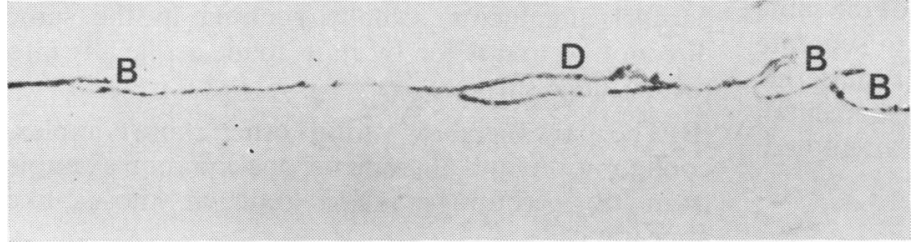

(16)

FIG S 14, 15, 16, 17 Vertically-cut histological sections of fixed tissue. Microscopic fields showing 'minimal' fibrillation. The sections were cut transverse to the predominant alignment of the markings seen when the surfaces were examined en face. Minute sharply-angled irregularities $(A)$ of the line of surface pigment; splitting $(B)$ extending tangentially immediately beneath the surface; formation of partly loose pieces of tissue $(C)$; and cavity $(D)$ containing Indian ink. $\times 150$

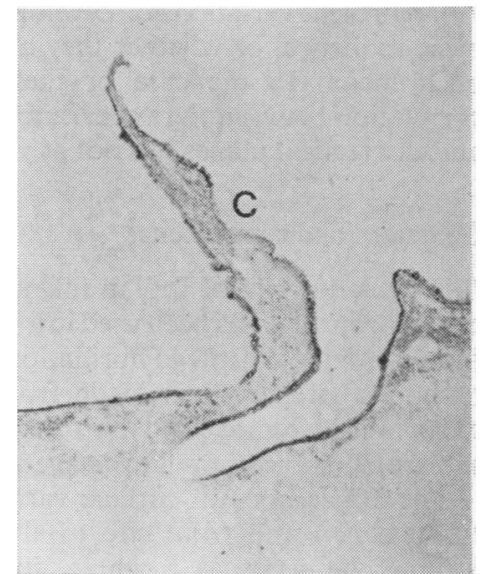

(17)

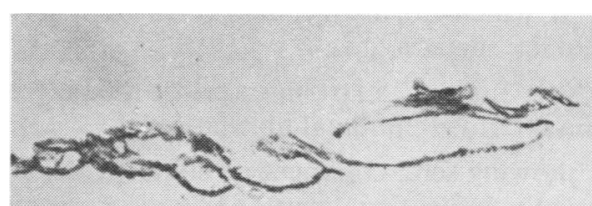

F I G S 18, 19 Examples of other surface patterns encountered in vertically-cut histological sections of 'minimal' fibrillation. Fixed tissue. $\times 150$

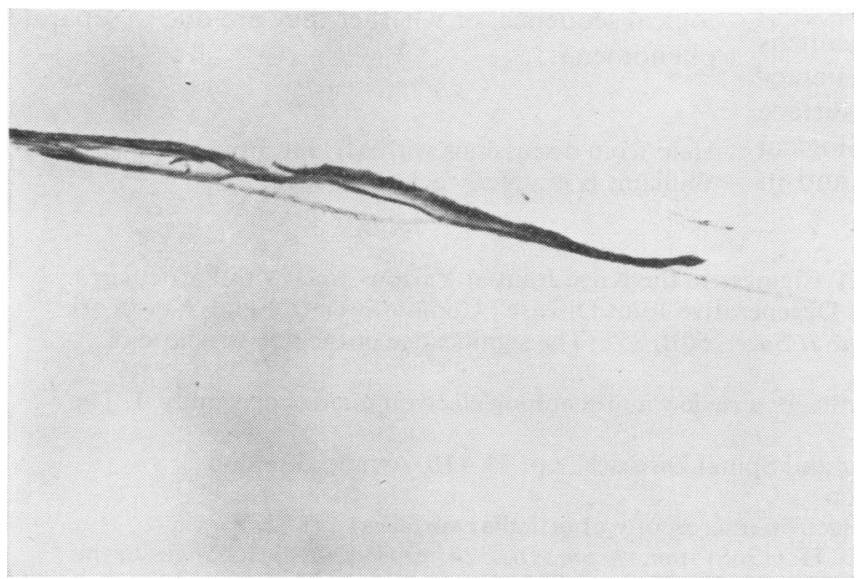

FIG. 20 Photomicrograph from one of a set of serial sections, cut vertically in an alignment parallel to the long axis of a partly loose surface strand at a site of 'minimal fibrillation'. Compare with the transversely-aligned sections shown in Figs 15, 16, 17. 'Minimal fibrillation'. $\times 150$

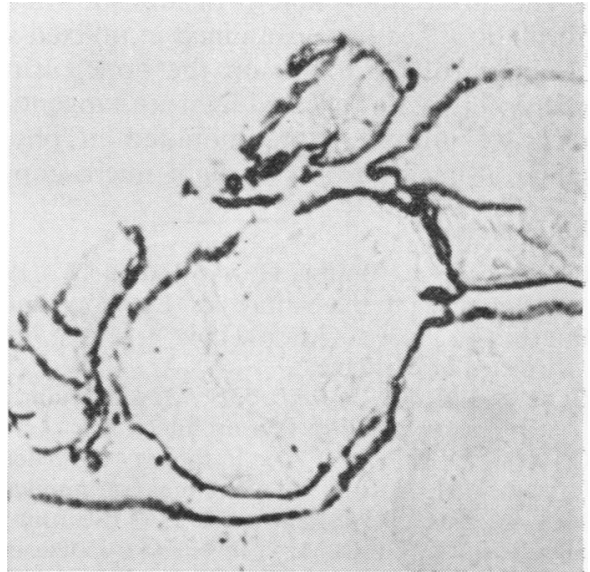

FIG. 21 Vertically-cut histological section showing overt fibrillation. Compare with Figs 15 to $20 . \times 150$ 
en face, and the sharply-defined grooves sometimes seen in this type of minimal fibrillation (Fig. 5) probably mark the site of rectangular strands which have peeled away from the surface. It is at present uncertain whether the other patterns encountered in tangential slices (Figs 11, 12) of minimal fibrillation, and other patterns seen in vertical sections (Figs 18, 19), represent variations or developments of the same basic sequence, or whether they are due to separate phenomena. In the case or these other patterns, correlation between the appearances in the tangential and the vertical planes has not as yet been attempted.

\section{Summary and conclusions}

Light microscopy of Indian ink preparations of the articular surface has been used in a study of the surface morphology of cartilage fibrillation. The findings are based on preparations made from patello-femoral articulations removed at necropsy in an unselected series of 29 subjects ranging in age from 18 to 91 years.

The appearance of surface varied from specimen to specimen and from site to site on the surface. Taking the series as a whole, four main types of surface appearance were encountered:

(1) Sites at which no distinct markings were seen, termed 'intact' surfaces;

(2) Sites showing dark markings against a pale grey background, termed 'minimal fibrillation';

(3) Sites showing semi-confluent or confluent blackening, usually indicating 'overt fibrillation' of the cartilage but sometimes due to an overgrowth of fibrous tissue at the extreme edge of the joint;

(4) Sites of bone exposure.

The surface morphology of sites showing 'minimal fibrillation' has been examined in unfixed specimens of the cartilage in situ on the bone, using stereomicroscopy with reflected light; in tangential surface slices of unfixed tissue mounted in physiological saline, using transmission light microscopy; and in histological sections of formalin-fixed cartilage. The findings indicate that an area of minimal fibrillation of the cartilage can exhibit a variety of surface patterns when examined en face in the tangential plane. Two main groups of patterns can be recognized on patellofemoral articulations:

(A) The Indian ink markings show approximately parallel alignments, usually running in the same direction as that taken by splits made artificially into the cartilage surface;

(B) The markings show various other, more complex, configurations and alignments, one common example from this group having a reticular mosaic-like appearance.

It is suggested that one way in which the state of "minimal fibrillation" may be initiated is from the site of a feature seen in vertical sections at a magnification of $\times 150$ as a minute, sharply-angled irregularity of the line of surface pigment, and on tangential slices at the same magnification, as fine, dark markings due to local concentrations of Indian ink particles. Tangential splitting can develop just beneath the surface from one edge of such a feature, giving broader markings in tangential slices; pieces of cartilage thus become free along one or more of their edges but elsewhere remain in continuity with the rest of the tissue; and partly loosened flakes and strands can form in this manner. This sequence of events will account for surface patterns of the type in which the markings show approximately parallel alignments when viewed en face. It is at present uncertain whether the other patterns encountered in tangential slices, and other patterns seen in vertical sections of 'minimal' fibrillation, represent variations or developments of the same basic histological sequence, or whether they are due to separate phenomena.

Help from discussions with Mr. Ian Emery and Dr. D. F. Williams is gratefully acknowledged.

\section{References}

Bennett, G. A., Waine, H. And Bauer, W. (1942) 'Changes in the Knee Joint at Various Ages with Particular Reference to the Nature and Development of Degenerative Joint Disease'. Commonwealth Fund, New York

Bullough, P., AND Goodfellow, J. (1968) J. Bone Jt Surg., 50B, 852 (The significance of the fine structure of articular cartilage)

Clarke, I. C. (1971) Ibid., 53B, 732 (Articular cartilage: a review and scanning electron microscope study. I. The inter-territorial fibrillar architecture)

Collins, D. H. (1949) 'The Pathology of Articular and Spinal Diseases', pp. 74-115. Arnold, London

Freeman, M. A. R. (1971) Personal communication

MCCALL, J. G. (1968) Lancet, 2, 1194 (Scanning electron microscopy of articular surfaces)

Meachim, G., Ghadially, F. N., ANd Collins, D. H. (1965) Ann. rheum. Dis., 24, 23 (Regressive changes in the superficial layer of human articular cartilage)

- AND RoY, S. (1969) J. Bone Jt Surg., 51B, 529 (Surface ultrastructure of mature adult human articular cartilage)

REDLER, I., AND ZIMNY, M. L. (1970) Ibid., 52A, 1395 (Scanning electron microscopy of normal and abnormal articular cartilage and synovium)

Walker, P. S., Sikorski, J., Dowson, D., Longfield, M. D., Wright, V., ANd Buckley, T. (1969) Ann. rheum. Dis., 28, 1 (Behaviour of synovial fluid on surfaces of articular cartilage. A scanning electron microscope study) 